\title{
Study on Mechanics Performance of Flexible Spacecraft Cables Combined with Complicated Working Conditions
}

\author{
Bindi You, Jianmin Wen*, Tianjiao Zheng, Chuansong Zhai, Binjiu Yang and \\ Guangbin $\mathrm{Yu}$ \\ (School of Naval Architecture and Ocean Engineering, Harbin Institute of Technology, \\ Weihai 264209, China) \\ *Corresponding author: Jianmin Wen; E-mail: wenjm@hit.edu.cn
}

\begin{abstract}
Taking the large deflection of flexible cables used in spacecrafts into ac ount a method is proposed to describe the position of any point on the cable using sagittal diameter, pitch angle, and deflection angle. Furthermore, the spatial attitude of the cable is cetermined using the mutual conversion of four coordinate systems. On the basis of the above a mechanics model that comprehensively considers physical factors, uch as mass, mechanical properties, and space constraints, is proposed in this paper. The forces and spatial attitudes of the equilibrium of the cable are also analyzed. In addition, Simulation andises of the proposed mechanics model are performed. Results are in reasonable agreement with the actual assembly environment.
\end{abstract}

Keywords: Flexible cable, Mechấnics modeling Environmental constraints, Simulation analysis

\section{Introduction}

Flexible cables are important components in mechanical and electrical products for satellites. Cables inside the satellite are mainly used to ensure signal transmission and communication between satellites and the ground. Moreover, these cables help transfer energy for all components. According to statistics, cables and power sources may account for $20 \%$ to $30 \%$ of the total weight of a satellite because of their complexity and variety [1]. Complex structures and unreasonable cable planning can result in a number of problems, such as chaotic structures, interferences, and poor communications [2]. Furthermore, the cost of launching is restricted by the weight of the atelite. However, the quality optimization of the satellite structure or other components is increasingly difficult to achieve. Therefore, we aim to develop mathematical models that can be used to reliably predict the dynamic performance of cables inside satellites, and to provide a theoretical basis for reasonable path planning.

Cables have flexible bodies with large deflection, complex shapes, a variety of types, and limited assembly space. Meanwhile, Interferences, electrical interfaces, the physical properties of the cable, and other factors need be considered in the assembly process. Thus, the basis of cable wiring is cable modeling, which includes the constraints of physical properties. Although certain CAD software currently has the corresponding cable module, the cable is not treated as a flexible deformation in this software. The mass, bending strength, tensile strength, and other mechanical properties of the cable cannot be reflected. Therefore, this software cannot meet the required authenticity in cable planning simulation. Based on the physical characteristics of cable, German scholar Hergenrother proposed the virtual cable [3]. Loock and Rabaetje proposed a mass-spring model $[4,5]$. American scholar Quisenberry put forward the lumped 
mass discretization model [6]. However, these models mainly use discretization to adjust cable position by controlling points and fitting the cable geometry is difficult. Accordingly, cable models that can satisfy the geometry and physical properties of the cable at the same time need to be established.

For the above, based on the flexible body of the cable, and considering winding, twisting, and other spatial attitudes of the cable, an appropriate method of expressing the spatial attitude of the flexible cable is proposed, and the equilibrium equations of the cable are established, in this study. Simulation calculations of the model are then performed, and the length, mass, and other parameters of the cable are predicted in order to replace the traditional simulation process involving artificial wood mold cabling. These methods can form a theoretical foundation for subsequent path planning studies and provide references for other flexible parts modeling.

\section{Establishment of Basic Space Coordinate Systems}

As shown in Figure 1, four coordinate systems are established. 1) Fixed nertial coordinate system: fixed coordinate system $\mathrm{O}-\mathrm{XYZ}$ is established at an atbitrary point $Q$ in the assembly space of the cables. 2) Position and posture coordinate system: the cable atutude is described by the coordinate system $P-\alpha \beta \gamma$, which is established at an arbitrary point $P$ on the cable, where direction $\gamma$ is the tangential direction of the cable, direction $\alpha$ is the normal direction of the cable at point $P$, and directions $\alpha, \beta$, and $\gamma$ are orthogonal. 3) Arc coordinate system s along the center line of the cable. 4) Local coordinate system $P-x y z$ established at point $P$ and is formed by the position and posture coordinate ystem with a rotation angle $\varphi_{l}$ around the axis $\gamma$.

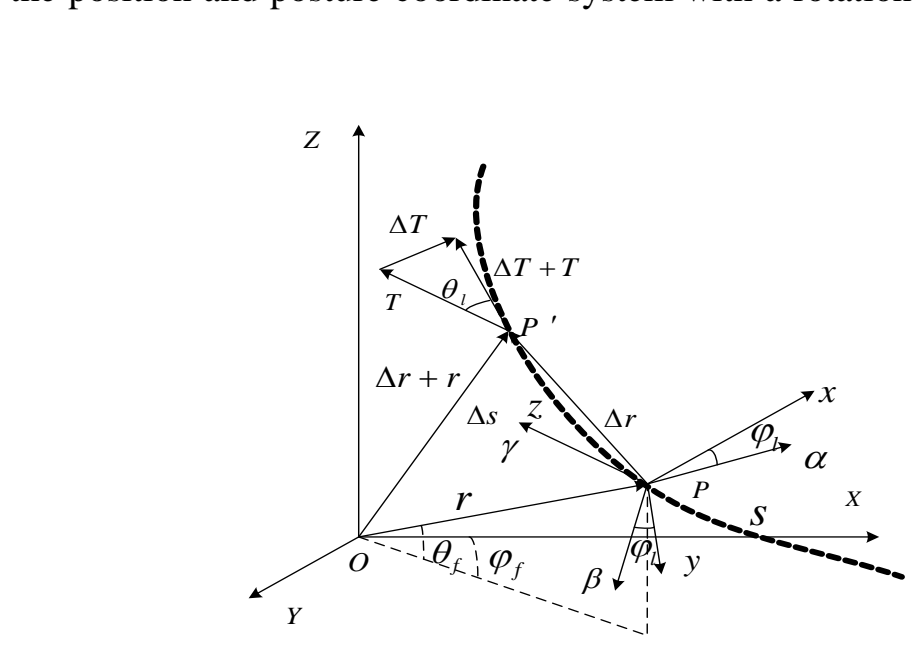

Figure 1. Basic Space Basic Coordinate Systems

\section{Expressions of Spatial Position and Posture of Flexible Cable}

To better express the spatial position and posture of the cable, the following idealized assumptions are made [7]:

1) Cross sections of the cable are regular in shape, and do not change with time and cable length in this study.

2) The center line of the cable is smooth and continuous, and the cable has a certain length.

3) Tension and shear deformation of the cable caused by forces are ignored; the cross section and center line are always orthogonal. 
4) The torsion of the cross section around the center line can be expressed by a continuous function.

After establishing the coordinate systems, the position of arbitrary point $P$ on the cable needs be determined in the fixed coordinate system. If the sagittal diameter is $r, R=|r|$, the pitch angle is $\theta_{f}$, and the deflection angle is $\varphi_{f}$, then the position of this point can be expressed as:

$$
r=\left\{\begin{array}{c}
X_{p} \\
Y_{p} \\
Z_{p}
\end{array}\right]=\left\{\begin{array}{c}
\cos \theta_{f} \cos \varphi_{f} \\
\cos \theta_{f} \sin \varphi_{f} \\
\sin \theta_{f}
\end{array}\right\rfloor R
$$

where $\theta_{f}, \varphi_{f}$, and $R$ are the single-valued function of $s$.

After the arc coordinate system and the position and posture coordinate system are established at point $P$, direction $\gamma$ is taken as the tangential direction of the cable The rotation angle of the cross section of the cable around the center line is $\varphi_{l}$, and the local bending angle of the cable is $\theta_{l}$. The curvature $\kappa$ and deflection $\tau$ are defined as follows:

$$
\kappa=|\dot{\gamma}|=\lim _{\Delta s \rightarrow 0} \frac{\Delta \theta_{l}}{\Delta s}=\dot{\theta}_{1}=|\beta|
$$

According to the definitions of the curvature, deflection, and the attitude coordinate system, deriving each coordinate axis of the attitude coordinate sysem relative to the arc coordinate system is performed as follows:

where

$$
\left(\frac{d \alpha}{d s} \quad \frac{d \beta}{d s} \frac{d \gamma}{d s}\right)=A(\alpha, \beta \gamma)^{T}
$$

is the transformation matrix between the position and posture coordinate system and the arc coordinate system.

In the position and posture coordinate system, the curvature and deflection of the cable are two independent variables that determine the spatial position and posture of the cable, respectively. Thus, the direction vectors of the center line of the cable in the position and posture coordinate system can be clearly expressed by the curvature and deflection of the cable. The spatial attitude of the cable can then be completely determined using the defining variation law of torsion angle $\varphi_{l}(s)$.

The motion of cable can be regarded as the motion synthesis of its rigid section along and aroun the center line. Therefore, the spindle coordinate system of section $(P-x y z)$ is established in this rigid section. In this coordinate system base, the vectors of the coordinate axes are $x, y$, and . Axis $z$ coincides with the tangential direction of the cable $\gamma$, that is, $z=\gamma$. In addition, the rotation angle of the section relative to the position and posture coordinate system is $\varphi_{l}$. Hence, the relationship between $(P-\alpha \beta \gamma)$ and $(P-x y z)$ is expressed as:

$$
\left(\begin{array}{lll}
\alpha & \beta & \gamma
\end{array}\right)^{T}=B\left(\begin{array}{lll}
x & y & z
\end{array}\right)^{T}
$$


where

$$
\boldsymbol{B}=\left(\begin{array}{ccc}
\cos \varphi_{l} & -\sin \varphi_{l} & 0 \\
\sin \varphi_{l} & \cos \varphi_{l} & 0 \\
0 & 0 & 1
\end{array}\right)
$$

is the transformation matrix between the position and posture coordinate system and the local coordinate system.

The above formulae show that the spatial position and posture of the cable in the local coordinate system can be determined using the curvature, deflection, and torsion angle.

Let the included angle cosine of the coordinate axes between the local coordinate system and the fixed coordinate system be $a, b$, and $c$, respectively. Thus, the conversion relationship between these two coordinate systems can be expressed as:

where

$$
\left[\begin{array}{llll}
X & Y & Z & 1
\end{array}\right]^{T}=\boldsymbol{C}\left[\begin{array}{llll}
x & y & z & 1
\end{array}\right]^{T}
$$

Until then, the position of an arbitrary point on the cable in the fixed coordinate system is expressed. The arc coordinate system, local coordinatesystem, and the position and posture coordinate system are established at this point. Meanwhile, the mutual conversion of these four coordinate systems can be realized using $\boldsymbol{A}, \boldsymbol{B}$, and $\boldsymbol{C}$. Thus, the spatial position and posture of the cable is expressed clearly.

\section{Mechanics Model of the Cable Considering Physical Properties}

As shown in Figure 2, to establish the mechanics equations, an arbitrary section of the cable is taken as the analysis object, and mechanics analyses are applied to the coordinate systems

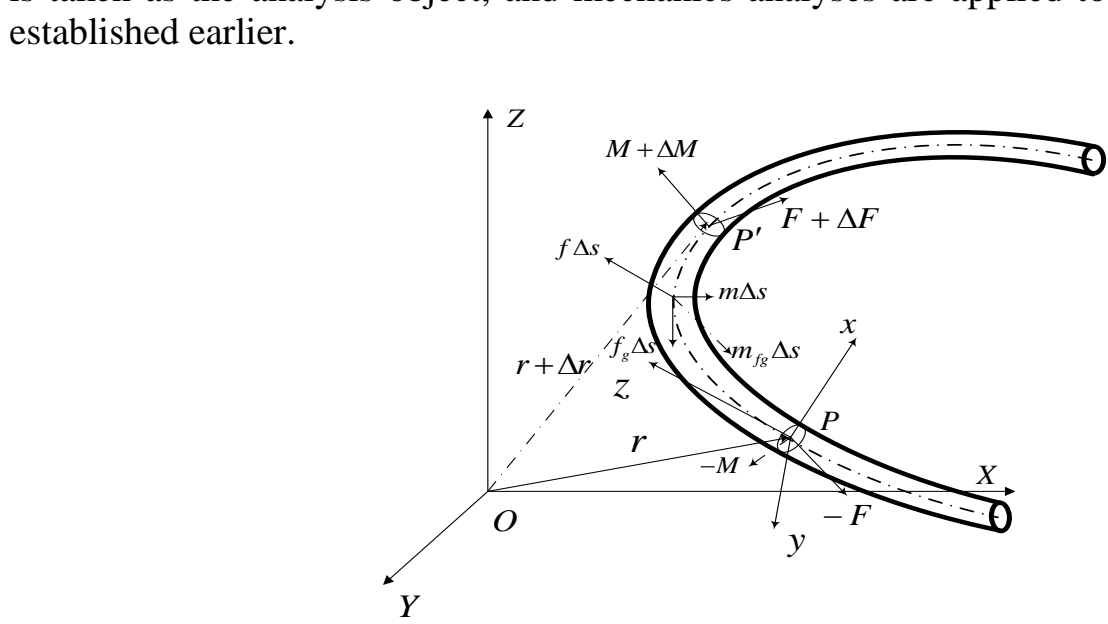

Figure 2. Mechanics Model of the Cable

Arbitrary motion of the cable is performed under the given forces and torques, where the degree of bending and torsion is $\omega$ at arbitrary point $P$. The density $\rho$ and section area $S$ of 
the cable are also defined. At point $P$, the principal vector and principal torque of the negative section with the internal force of the adjacent section are $-F$ and $-M$, and are $(F+\Delta F)$ and ( $M+\Delta M$ ), respectively, at the adjacent point $P^{\prime}$. In addition, the environmental force and environmental torque of the cable per unit length are $f$ and $m$, and the force and torque caused by deadweight of the cable per unit length are $f_{g}$ and $m_{f g}$, respectively.

When the point $P$ has a positive movement with unit velocity along the arc coordinate $s$, the section rotates relative to the fixed coordinate system $\mathrm{O}-X Y Z$ with $\omega$. Therefore, the degree of bending and torsion $\omega$ is defined as [8]:

$$
\omega=\kappa \beta+\tau \gamma+\frac{d \varphi_{l}}{d s} z=\omega_{x}+\omega_{y}+\omega_{z}
$$

where

$$
\omega_{x}=\kappa \sin \varphi_{l}, \quad \omega_{y}=\kappa \cos \varphi_{l}, \quad \omega_{z}=\tau+\frac{d \varphi_{l}}{d s}
$$

are the components of the degree of bending and torsion in every direction of the local coordinate system.

The force conditions of the cable when it is in a balanced and static state are shown in Figure 2. According to Newton's second law and the Momentum Theorem, we obtain

$$
\left\{\begin{array}{l}
\Delta F+f \Delta s+f_{B} s=0 \\
\Delta M+m \Delta s-m_{f g} \Delta s+\Delta Q j=0
\end{array}\right.
$$

If both sides of Equations (11) are divided by $\Delta s$, and let $\Delta s \rightarrow 0$, then

After the paranneters are derived from the local coordinate system $p$-xyz, according to the relationship between the absofute derivative and the relative derivative, we obtain

$$
\left\{\begin{array}{l}
\frac{\tilde{\partial} F}{\partial s}+\omega \times F+f+f_{g}=0 \\
\tilde{\partial} M \\
\frac{\tilde{\partial} s}{\partial s}+\omega \times M+m+m_{f g}+z \times F=0
\end{array}\right.
$$

The projections of Equations (13) on the coordinate axes are shown as:

$$
\left\{\begin{array}{l}
\frac{\partial F_{x}}{\partial s}+\omega_{y} F_{z}-\omega_{z} F_{y}+f_{x}+f_{g x}=0 \\
\frac{\partial F_{y}}{\partial s}+\omega_{z} F_{x}-\omega_{x} F_{z}+f_{y}+f_{g y}=0 \\
\frac{\partial F_{z}}{\partial s}+\omega_{x} F_{y}-\omega_{y} F_{x}+f_{z}+f_{g z}=0
\end{array}\right.
$$




$$
\left\{\begin{array}{l}
\frac{\partial M_{x}}{\partial s}+\omega_{y} M_{z}-\omega_{z} M_{y}+m_{x}+m_{f g x}-F_{y}=0 \\
\frac{\partial M_{y}}{\partial s}+\omega_{z} M_{x}-\omega_{x} M_{z}+m_{y}+m_{f g y}+F_{x}=0 \\
\frac{\partial M_{z}}{\partial s}+\omega_{x} M_{y}-\omega_{y} M_{x}+m_{z}+m_{f g z}=0
\end{array}\right.
$$

where

$$
\left\{\begin{array}{l}
M_{x}=E I_{x}\left(\omega_{x}-\omega_{x}^{p}\right) \\
M_{y}=E I_{y}\left(\omega_{y}-\omega_{y}^{p}\right) \\
M_{z}=G I_{z}\left(\omega_{z}-\omega_{z}^{p}\right)
\end{array}\right.
$$

$E$ and $G$ denote elastic modulus and shear modulus, respectively.

Substitute in Equations (15), then

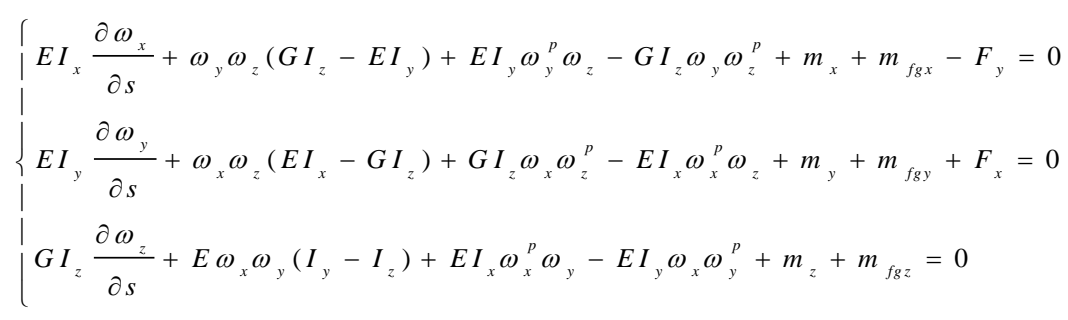

The equilibrium equations of the cable can be óbtained by simultaneously using Equations (14) and (17), where $F$ and $\omega$ are unknown yariables, and $G, E, f, f_{g}, I, m$, and $m_{f g}$ are known variables. Therefore, the balance equations are solvable.

\section{Simulation Examples}

Focusing on the copper cable whose section area is $10 \mathrm{~mm}^{2}$, When the curvature and the deflection of the cable are defined, the variation law of the direction vectors in the position and posture coordinate system canbe obtained using equation (3).

Let the curvature and deflection be 1 , then a numerical integration is performed by using Runge-Kutta. When the initial coordinate of the cable is $(1,1,1)$, the variation law of the direction vectors in the position and posture coordinate system is obtained as shown in a) of Fig. 3. When the curvature and deflection of the cable are fixed values, the spatial attitude of the cable center line shows a spatial circle in the attitude coordinate system.

Likewise, let the length of the cable be 10 and the initial coordinate of the cable be $(1,1,1)$. Several-groups of the spatial attitude coordinates of the cable can be obtained when different curvatures and deflections are taken from Table 1. Furthermore, the length of the cable with the sane terative steps is obtained in each group. The spatial attitude coordinates of the cable with variable curvature and deflection are established. The simulation results of the cable obtained using visualization processing of the data are shown in b) of Figure 3.

Table 1. Curvature and Deflection

\begin{tabular}{cccccccccc}
\hline$\kappa$ & 0 & 0.1 & 0.5 & 1 & 1 & 5 & 2 & 0.7 & 0.9 \\
\hline$\tau$ & 0 & 5 & 2 & 1 & 0.7 & 0.1 & 0.5 & 1 & 0.8 \\
\hline
\end{tabular}


When the curvature and deflection of the cable are with irregular variations along the cable, the attitude variations of the cable in the position and posture coordinate system are also irregular. When the torsion of the cable is not considered, the attitude of the cable is completely determined using the curvature and deflection of the cable. Bending deformations of the cable with external-force constraints are produced in many inflection points on the cable to adapt to the complex assembly space of the cable. Moreover, the curvature and deflection of the cable between the inflection points are constant.

Considering the properties of the cable section and the rotations of the cable around the center line, when the torsion angle is changed uniformly, the coordinates of the position and posture coordinate system are transformed by means of transformation matrix $\boldsymbol{B}$. The simulation results of the cable in the local coordinate system are shown in a) of Figure 4 . The shape of the cable does not vary after the spatial attitude has transformed. And, the whole cable merely deflects around the coordinate axis. The simulation results coincide with the mathematical expressions.

The attitude of the cable in the fixed coordinate system can be obtained from the local coordinate system by transformation matrix $\boldsymbol{C}$. The local coprdinate system is obtained by rotating the fixed coordinate system using the variation of the Euler angle and the translation between point $P$ and the fixed point. Thus, if the values of the Euler angle range from 0 to $2 \pi$ with uniform change, the attitude of the cable in the fixed coordinate system can be obtained, as shown in b) of Figure 4.

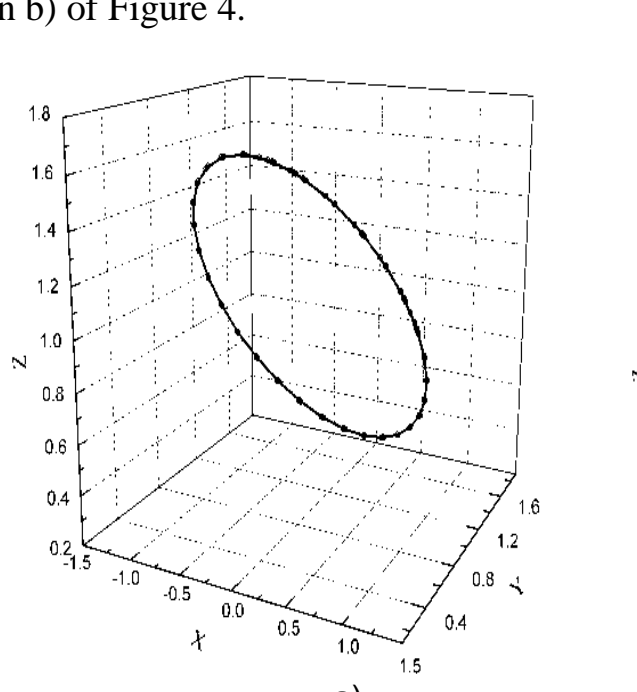

a)

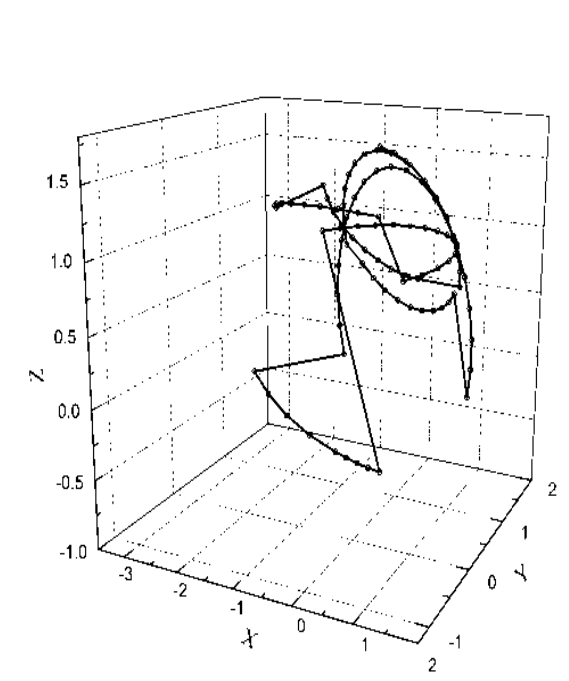

b)

\section{Figure 3. a) Cable Position and Posture with Fixed Curvature and Deflection;b) Attitude of Cable in the Position and Posture Coordinate System}

The cable attitude greatly changes in the fixed coordinate system because of the angle relationship between coordinate axes of the fixed coordinate system and the local coordinate system of the cable. From the view of operator, the attitude of the cable can be better controlled in real-time and the cable planning can be better realized because the fixed coordinate system is fixed in the assembly space. 


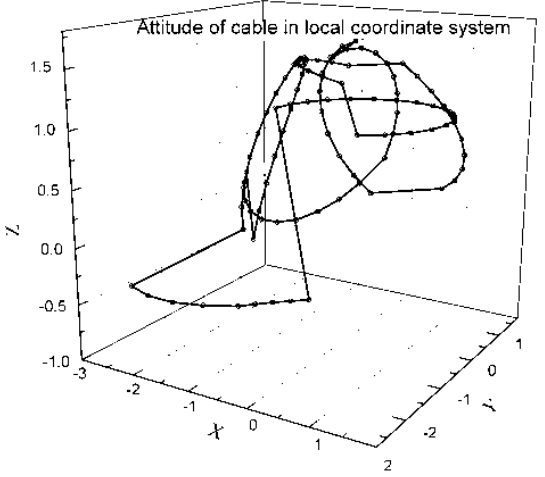

a)

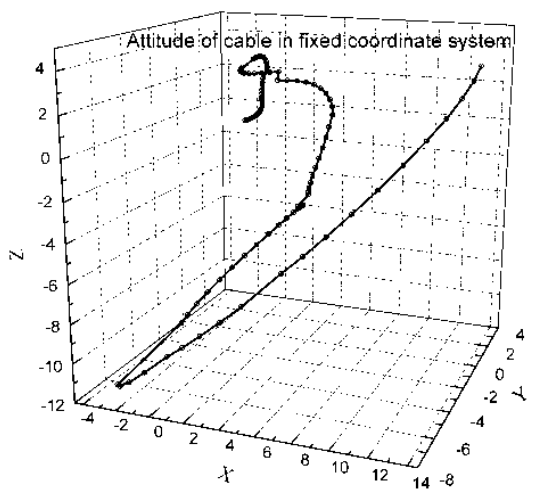

b)

Figure 4 a) Attitude of Cable in the Local Coordinate System; b) Attitude of Cable in Fixed Coordinate System

The spatial attitude of the cable is maintained in the static ctate by the internal force of the cable, and its mathematical model is given by Equation (11). Given the initial deadweight force, deadweight torque, environmental distributed force, and environmental distributed torque, then the proposed model is solved by using Runge-Kutta. Hence, variation laws of the internal force and degree of bending and torsion of the cable are obtained.

Physical properties and environment of the cable are considered in the establishment of the mechanics model. The initial force condition of the cable are shown in Table 2.

Table 2. Initial Force Conditions of the Cable

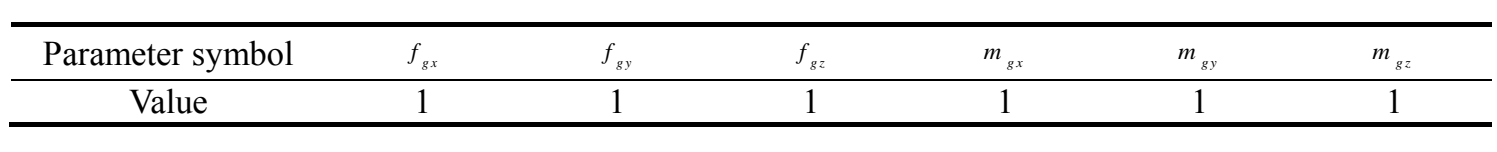

According to the equilibrilm conditions of the cable, variation laws of the force and the degree of bending and torsion of the cable are calculated and shown in Figure 5.

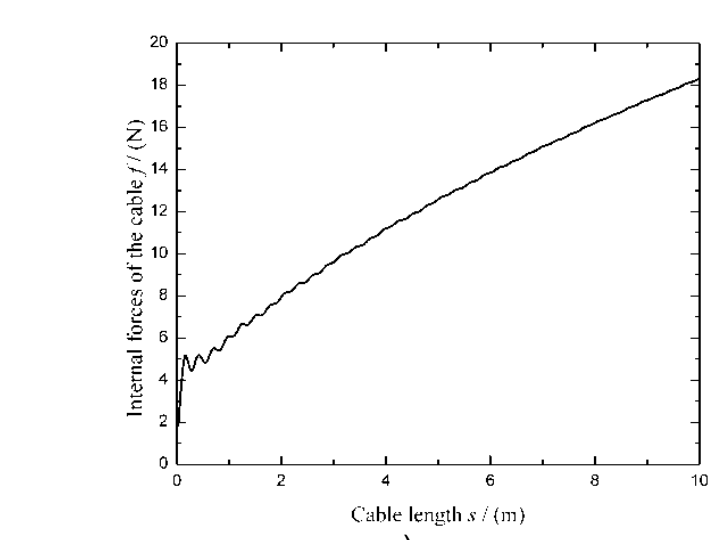

a)

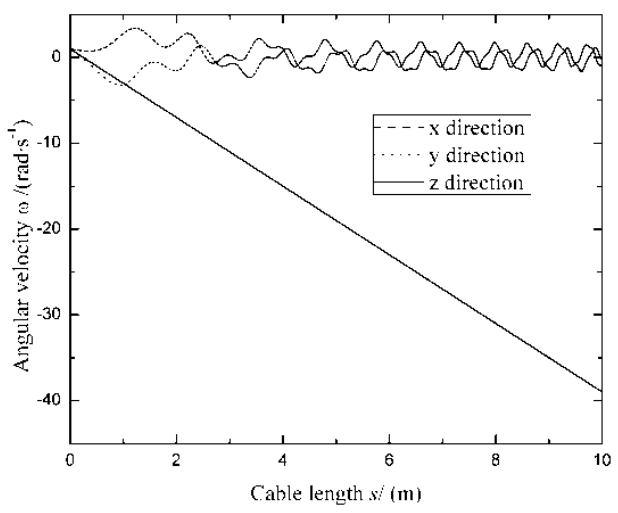

b)

Figure 5. a) Internal Force of the Cable in the Equilibrium State, b) Degree of Bending and Torsion of the Cable in the Equilibrium State

The results show that to maintain the balance of the cable, the steady improvement trend with concussion of the force is presented with the increase in the length of the cable. The value of the initial force is not 0 in the origin because of the deadweight force and the spatial 
constraint forces. The trend of the forepart of the curve has small amplitude fluctuations, and then gradually stabilizes at the latter part of the curve.

The degrees of bending and torsion in directions $x$ and $y$ are stable with constant amplitude concussions. And, the degree of bending and torsion in the direction $z$ has a linear relationship with the length of the cable. These results occur because that to maintain the equilibrium state, the torque of the cable is achieved using the component in direction $z$.

Upon obtaining the degree of bending and torsion of the cable, the spatial attitude of the cable can be obtained using the relationships between the bending and torsion and the curvature, deflection, and torsion angle of the cable. Hence, the spatial attitude of the cable with the specific constraints can be obtained.

\section{Conclusions}

Taking the requirements for cabling into account, mechanics models that consider the physical properties of the cable are proposed. The variation laws of the spatial attitude, the motion parameters, and the internal force of the cable with constraints are analyzed in this study.

1) The elastica equations of the cable are determined by using sagittal diameter, deflection angle, and pitch angle. Moreover, the spatial attilude of the cable is expressed by mutual conversion of four coordinate systems: fixed coordinate, position and posture coordinate, local coordinate, and arc coordinate systems. Three degrees of freedom, namely, curvature, deflection, and torsion angles, are then deternimed.

2) Static equilibrium model of the cable is established. Upon selecting the spatial constraints and initial conditions of the cable, the yariation lays of the cable parameters are obtained using a numerical method. The majority of the internal forces and degrees of bending and torsion of the cable are observed in the axiadirection. Hence, these results are consistent with the requirements of actual cabling.

\section{Acknowledgements}

This paper is based on Projects (Approval No. 51205079 and 51275108) Supported by National Natural science Foundation of China, and Project (HIT.KLOF.01507374) Supported by Key Laboratory Opening Funding of Technology of Micro-Spacecraft, Project (HIT. NSRIF. 2015110) suppopted by Natural Scientific Research Innovation Foundation in Harbin Institute of Technology, and China Postdoctoral Science Foundation funded project (2013M541358), and the Key Program of National Natural Science Foundation of Heilongjiang (No.ZD201309), and the Major International Joint Research Program of China (Grant No. 2014DFB70120). The authors would like to thank the research grant from Spacecraft Dynamics Design and Simulation Lab (SDDSL) of HIT.

\section{References}

[1] V. A. Emil and V. Babuška, "Dynamics of cable harnesses on large precision structures. 48th AIAA/ASME/ASCE/AHS/ASC Structures", Structural Dynamics, and Materials Conference, (2007), pp.1-11.

[2] J. M. Ritchieand and G. Robinson, "Cable harness design, assembly and installation planning using immersive virtual reality”, Virtual Reality, (2007), pp.261-273.

[3] E. Hergenrother and P. Dahne, "Real-time virtual cables based on kinematic simulation", Proceedings of the WSCG, (2000), pp.402-409.

[4] A. Loock and E. Schomer, "A virtual environment for interactive assembly simulation: from rigid bodies to deformable cables", 5th World Multiconference on Systemics, Cybernetics and Informatics (SCI'01), (2001), pp.325-332. 
[5] R. Rabaetje, "Real-time simulation of deformable objects for assembly simulation", Proceedings of the 4th Australian User Interface Conference on User Interfaces, (2003); Adelaide, Australia, pp.57-64.

[6] J. E. Quisenberry and A. S. Arena, "Discrete cable modeling and dynamic analysis", 44th AIAA Aerospace Sciences Meeting and Exhibit, (2006); Reno, Nevada, pp.1-16.

[7] Y. Xue, Y. Z. Liu and L. Q. Chen, "On analytical mechanics for a super-thin elastic rod", Chinese Journal of Theoretical and Applied Mechanics, vol. 4, no. 37, (2005), pp.485-493.

[8] Y. Xue, Y. Z. Liu and L. Q. Chen, "Methods of analytical mechanics for dynamics of the Kirchhoff elastic rod”, Acta Physica Sinica, vol. 8, no. 55 (2006), pp.3845-3851.

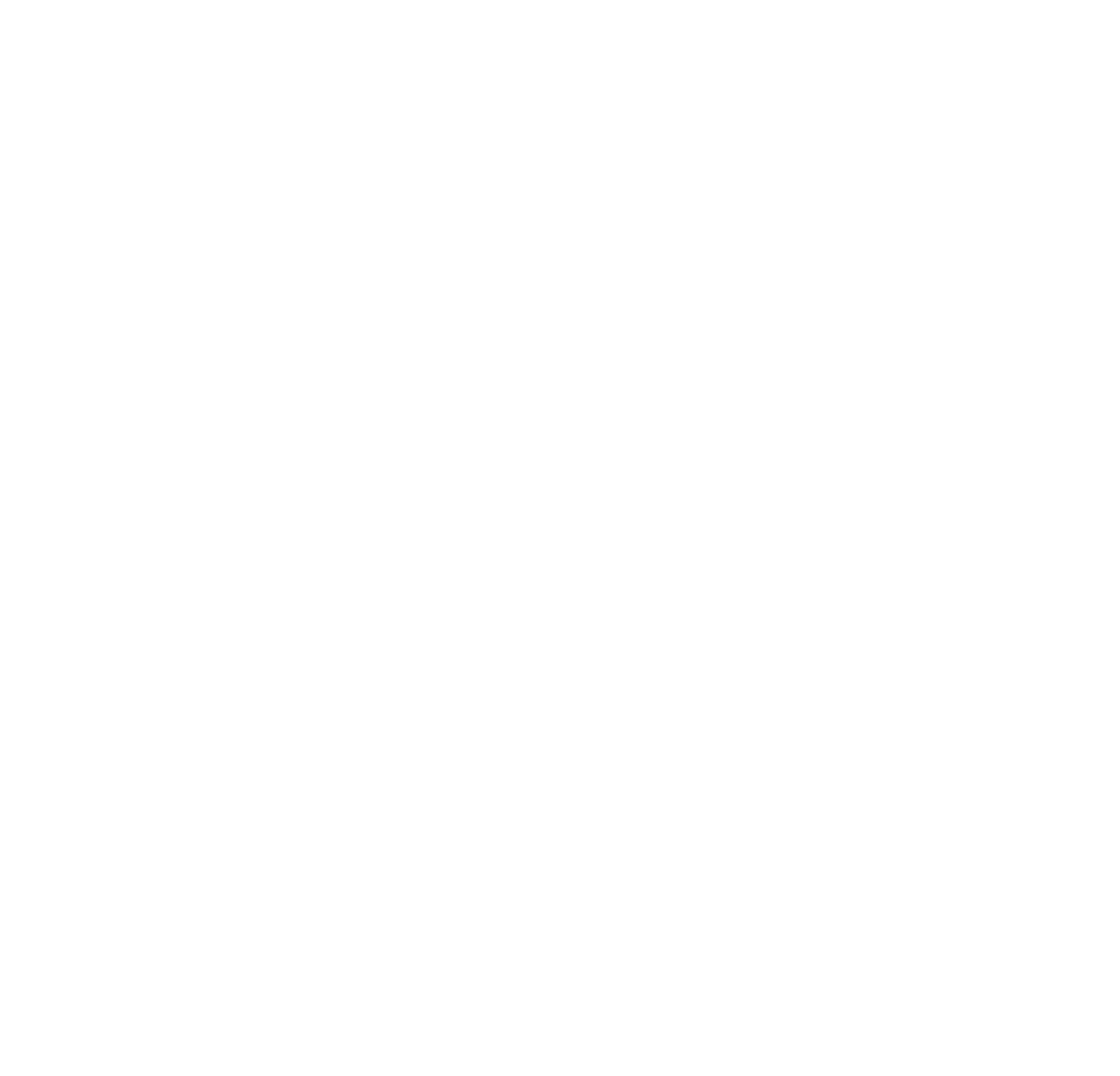

